\title{
Thoracic surgery in Hong Kong
}

\author{
Aliss T. C. Chang^, Joyce W. Y. Chan, Rainbow W. H. Lau, Calvin S. H. Ng \\ Division of Cardiothoracic Surgery, Department of Surgery, Prince of Wales Hospital, the Chinese University of Hong Kong, Hong Kong, China \\ Contributions: (I) Conception and design: CSH Ng, JWY Chan; (II) Administrative support: CSH Ng, RWH Lau, JWY Chan; (III) Provision of study \\ materials or patients: JWY Chan, CSH Ng, RWH Lau; (IV) Collection and assembly of data: All authors; (V) Data analysis and interpretation: All \\ authors; (VI) Manuscript writing: All authors; (VII) Final approval of manuscript: All authors. \\ Correspondence to: Dr. Calvin S. H. Ng, MD, FRCS. Professor, Division of Cardiothoracic Surgery, Department of Surgery, The Chinese University \\ of Hong Kong, Prince of Wales Hospital, Hong Kong, China. Email: calvinng@surgery.cuhk.edu.hk.
}

\begin{abstract}
In the era of modernized medicine, thoracic surgery has been focusing on achieving minimally invasive surgery and providing a one-stop solution in treating thoracic diseases. Particularly in the Asia population, where patients are keen to have smaller wound and shorter hospital stay, thoracic surgery in Hong Kong has evolved from the traditional open thoracotomy approach to video-assisted thoracoscopic surgery (VATS). In our institution, uniportal VATS for major lung resection was developed in 2012. While uniportal VATS has brought advantages into managing thoracic pathologies, it also brought challenges like instrument fencing during manipulation and suboptimal visualization angle. To improve the procedure and its outcomes, novel techniques and equipment have been developed, for example, double-hinged instruments, robotic assisted technology and magnetic anchored and guided endoscopes (MAGS). With advanced medical imaging nowadays, management of small lung nodules or ground glass opacity (GGO) is in higher demand than ever before. Our hybrid operating room (HOR) can incorporate instant and real-time imaging in lesion localization, and provide treatment via VATS or electromagnetic navigated bronchoscopic (ENB) ablation in a one-stop manner. This paper will review the literature related to the historical development and clinical outcomes of thoracic surgery in Hong Kong and discuss the future perspective of ongoing development.
\end{abstract}

Keywords: Electromagnetic navigation bronchoscopy; hybrid operating room; lung cancer; uniportal VATS; robotic thoracic surgery

Submitted Oct 14, 2021. Accepted for publication Jan 06, 2022.

doi: $10.21037 /$ jtd-21-1600

View this article at: https://dx.doi.org/10.21037/jtd-21-1600

\section{Introduction}

Thoracic surgery in Hong Kong was a budding subspecialty back in the 1950s, focusing mainly on non-anatomical lung resection and treatment for pulmonary tuberculosis in open thoracotomy approach. As time passed, the spectrum of lung diseases changed, and new technologies emerged for both diagnosis and treatment. Currently, there are four thoracic surgical centers in the public sector which provide service to approximately 7.4 million Hong Kong residents, treating a wide range of thoracic diseases, including lung cancers, mediastinal tumors, chest wall tumors and deformities, pleural diseases, empyema, thoracic trauma, airway diseases and more. Lung cancer remains the most prevalent surgical lung disease in our locality. According to the Hong Kong Cancer Registry, lung cancer is the commonest cancer in Hong Kong and has the highest mortality rate among all cancer deaths. In 2019, 15.9\% of all cancer patients were diagnosed with lung cancer and $27.1 \%$ of cancer patients died from lung cancer (1). In 2019, there were 5,575 newly diagnosed cases of lung cancer (1). An early 2000s study concluded that direct inhalation or environment exposure to tobacco smoke and occupational fume exposure are two

$\wedge$ ORCID: 0000-0002-6672-5894. 
major risk factors associated with lung cancer in Hong Kong (2). With the increased availability of computed tomography (CT) scans of the thorax, more patients present with incidental finding of small lung nodules or ground glass opacities (GGO), creating challenges in both diagnosis and pre-operative localization, especially if they are deep, small and subsolid. Hence, major recent advancements on thoracic surgery aimed to tackle the changing demographics of thoracic malignancies. In this article, we shall review the evolution of thoracic surgery and technologies in the recent decades, and explore the future directions for thoracic surgical practice in Hong Kong.

\section{Development of video assisted thoracoscopic surgery (VATS) in Hong Kong}

Open thoracotomy was the preferred route of access in the first few decades of thoracic surgery development in Hong Kong. However, conventional thoracotomy is one of the most painful surgical incisions in the body, due to its long length, need of muscle splitting, and commonly required rib spreading or fracture. This leads to prolonged recovery and hospital stay, and severe wound pain which often persists for years as post-thoracotomy pain syndrome (3). Following the success of laparoscopic cholecystectomy in the 1980s which demonstrated reduced pain and faster recovery, video assisted thoracoscopic surgery was introduced in the early 1990s, and pioneers in Hong Kong were quick to explore and adopt the new technique. Professor Anthony Yim from The Chinese University of Hong Kong reported a total of 284 VATS procedures performed in 192 patients between 1992 and 1994, demonstrating that a wide range of thoracic procedures can be safely and effectively performed with VATS $(4,5)$. Since then, VATS rapidly became the de facto surgical technique in Hong Kong in tackling a broad range of intrathoracic illnesses, from diagnostic procedures such as wedge resection and pleural biopsy, to interventional procedures like surgical pleurodesis, major lung resection, mediastinal mass excision and more.

The routine technique in performing VATS in Hong Kong involve placing the patient in a full lateral decubitus position with flexion to open up the intercostal spaces; and double lumen endotracheal intubation with one lung ventilation. Ten mm 30-degree videoscope is commonly used and an exploratory thoracoscopy is customarily performed before proceeding to the main operation. The commonest form of VATS utilizes two to three ports and is usually without rib spreading in most cases. $\mathrm{CO}_{2}$ insufflation is not routinely used in our practice. Early result of VATS for major lung resection has been reported in 1998, concluding that VATS provide a favorable intermediate result in major lung resection patients with stage I to stage IIIA primary lung cancer, with $93 \%$ survival at 26 months of follow-up (5). In addition to the favorable oncological outcomes, VATS offers a better quality of life, as it resulted in less blood loss, shorter hospital stay, decreased immune disturbance and better preserved shoulder function when compared with open approach (6-9). Therefore, despite continual increase in the number of thoracic patients treated per year, the number of beds dedicated to thoracic surgery has risen proportionally less, due to a general trend of shorter length of stay and lower complication rates.

\section{Uniportal VATS}

In early 2000s, uniportal VATS was pioneered by several European groups, such as Gaetano Rocco's group in Italy (10) and Gonzalez Rivas's group in Spain (11). The first uniportal VATS for sympathectomy was reported back in 2002, first wedge resection in 2004, while uniportal lobectomy was not achieved until 2010 (12). Meta-analyses supported the use of uniportal VATS for treatment of lung cancer, demonstrating a significant reduction in overall rate of complications, length of hospital stay and duration of chest drain placement $(13,14)$. Hong Kong is one of the earliest Asian regions to embark on the journey of uniportal VATS development in the 2010s (15). This revolution from multiportal to uniportal VATS in Hong Kong has been accelerated when studies showed that uniportal VATS is associated with less early post-operative pain (Figure 1), reduced analgesic requirements (13) and decreased hospital stay $(6,15)$, without oncological compromise in terms of survival or the number of lymph nodes dissected (15). A recent study in 2021 also showed less immunochemokine disturbance in patients following uniportal VATS when compared with multiportal VATS (16).

Similar to overseas experience, our institute first attempted sympathectomy during our learning curve on single port VATS. We developed a novel approach utilizing the Vasoview Hemopro 2 endoscopic vein harvesting device (Getinge, Gothenburg, Sweden), which incorporated an endoscope with built-in working channel for a diathermy dissector, a retractable lens cleansing system and a $\mathrm{CO}_{2}$ insufflation channel (17). This allows effective thermoablation of the sympathetic ganglions and associated nerves, and allows a single surgeon to complete the procedure 


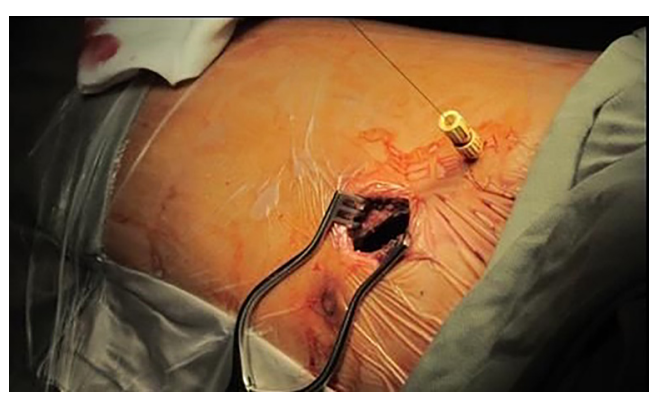

Figure 1 Hookwire localization of small lung lesion in the hybrid operating room, followed by same session VATS wedge resection. This reduces the risk of pneumothorax and hookwire dislodgement as minimal patient transfer is required. VATS, video-assisted thoracoscopic surgery.

by combining the videoscope and the dissector in one device. Uniportal lung wedge resections, pleurodesis and pericardial windows quickly followed suit (18).

Hong Kong's first uniportal VATS anatomical lung resection was performed by Professor Calvin $\mathrm{Ng}$ at Prince of Wales Hospital in 2012 (19). In March 2013, the first Asian Single Port VATS Symposium (ASPVS) was hosted, with live surgeries attended by speakers and attendees from all corners of the world. This symposium has since become a greatly anticipated annual event that has taken place all over Asia, and resulted in high quality scientific publications $(20,21)$. From our initial experience with 150 consecutive cases, uniportal VATS showed good safety profile, low peri-operative morbidities and mortality with zero 30-day mortality and 3\% 2-year overall mortality. Most of these patients (87\%) had non-small cell lung carcinoma (NSCLC), where uniportal VATS showed comparable disease-free survival rate to conventional VATS in both stage I (96\%) and stage II or above (83\%) disease (15).

Yet, reducing the invasiveness of VATS came at a price of increased technical challenge (15), especially in Asian population where thoracic cavity is smaller and intercostal spaces are narrower (22). Small single incision makes accommodation of videoscope and instruments difficult, and has less space for intra-operative maneuvers such as retraction and stapling. Fortunately, new instruments and technologies specifically designed to overcome these challenges were developed. We use angulated and narrowshafted instruments which have double hinged design to make room for maneuvering in the thoracic cavity and avoid instrument fencing. 5-mm 30-degree thoracoscope with combined light source and cable is usually used in the single port setting, allowing more instruments in a smaller wound, better unparalleled visualization angle, and less instrument fencing (23). The demands of uniportal VATS also provided opportunities for us to lead the development and testing of flexible and low-profile endostaplers to enable safe stapling in awkward angles (24). Furthermore, uniportal access drove the innovation of alternative lung resection technique other than by endostaplers, such as utilizing surgical lasers (25).

Interestingly, due to simultaneous development of uniportal VATS in many Asian countries, each VATS center has invented its own signature uniportal VATS approach based on its 3-port VATS experience. In Hong Kong, most centers prefer a 30 -degree 5 or $10 \mathrm{~mm}$ scope, while others may prefer a more versatile 120-degree larger thoracoscope or flexible tipped thoracoscope for vision (26). While some surgeons prefer to stand at the posterior side of patient and operate through a lateral port incision, we prefer to approach from the anterior and work through a utility port which is usually centered over the anterior axillary line to take advantage of the wider intercostal spaces (27).

\section{Electromagnetic navigation bronchoscopy (ENB): biopsy, dye marking and ablation in hybrid operating room (HOR)}

Recent years witnessed a drastic change in thoracic disease spectrum, from large lung tumours detected on chest X-rays, to small subsolid lung nodules incidentally discovered on CT scans (12). While smoking population in Hong Kong gradually shrinks thanks to public health education $(2,28)$, there is a rising proportion of female non-smoker lung cancer patients who often harbour adenocarcinomas instead of the squamous subtype according to local cancer registries. Similar to colon cancers, pulmonary adenocarcinomas have been shown to follow a step-wise progression from pre-malignant (atypical adenomatous hyperplasia) to malignant lesions (adenocarcinoma insitu, minimally invasive adenocarcinoma, and eventually invasive adenocarcinoma) (29). Some of these present as ground glass opacities on imaging that can be more aggressive in their behavior than previously thought (30). Hence, early treatment of these relatively small and subsolid lung nodules could provide an effective way to prevent development into cancer and may improve survival.

The challenges for this approach, though, would be accurate localization for diagnosis and subsequent treatment. The initial solution we had in 2014 at Prince 


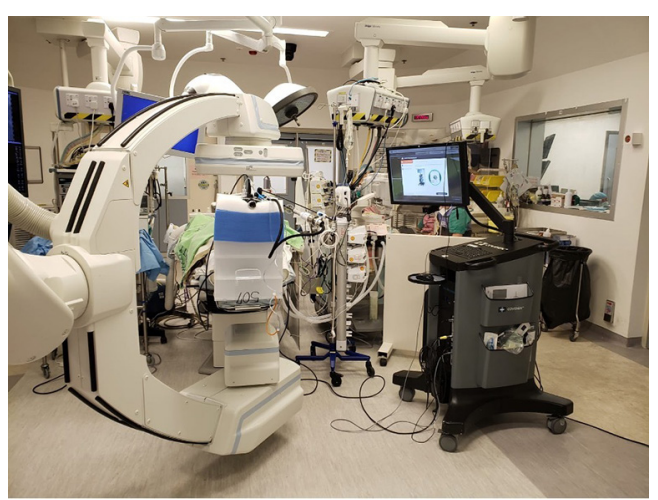

Figure 2 Usual set-up of our HOR using ENB, in combination with intra-operative cone-beam CT. HOR, hybrid operating room; ENB, electromagnetic navigation bronchoscopy; CT, computed tomography.

of Wales Hospital is the utilization of a hybrid operating room with cone-beam CT (CBCT) and fluoroscopy, which is magnetic and radiation compatible, to perform percutaneous hook-wire localization of small subsolid lung nodules followed by uniportal VATS resection in the same session (31) (Figure 2). This stream-lined workflow by avoiding the delay between localization and surgery improved patient experience and reduced complications such as pneumothorax and hookwire dislodgement, when compared with the conventional arrangement of hookwire insertion in the radiology department and subsequent transferal to the operating room (32).

However, this approach only provided a partial solution, thus in 2015, electromagnetic navigation bronchoscopy (ENB) was introduced into our hybrid theatre and rapidly became an important tool for managing small lung nodules (Figure 3). ENB utilizes electromagnetic positioning and specialized software, for example the SuperDimension ${ }^{\circledR}$ Navigation system (Medtronic, Minneapolis, USA), to analyze the pre-operative CT scan to create a $3 \mathrm{D}$ virtual roadmap of the airways, through which the bronchoscope can be accurately guided towards the target lesion. Combining the application of ENB with real time CBCT and fluoroscopic overlay within the hybrid theatre allows us to correct any misdirection and to confirm precise position of bronchoscopic tools. Through the working channel of the navigation bronchoscope, various advanced tools can be utilized to perform diagnostic and therapeutic procedures, such as biopsy, dye marking for subsequent resection, placement of fiducial markers, and ablation (33) (Figures 4,5).

From our experience and those of others, real-time multimodal imaging in HOR increases diagnostic yield

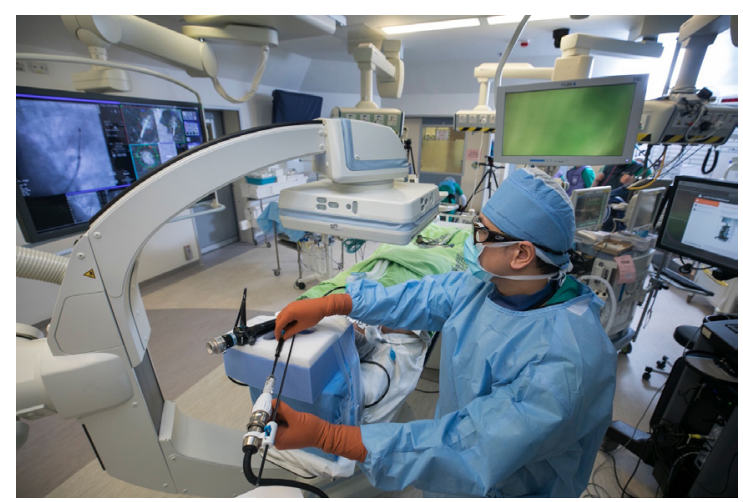

Figure 3 Navigation towards target lesion with ENB and fine realtime adjustments under fluoroscopy guidance. Eventually, position of the bronchoscopic tool was confirmed with CBCT. ENB, electromagnetic navigation bronchoscopy; CBCT, cone-beam computed tomography.

(34-36), particularly in small pulmonary lesions $<1 \mathrm{~cm}$ and GGO lesions, permits multi-instrument biopsy and allows multiple lesions to be biopsied in one session.

Furthermore, the HOR can provide a one-stop diagnostic, localization and therapeutic solution. For small lung lesions which may not be palpable during surgery, after biopsy was taken via ENB and diagnosis made by intra-operative frozen section, dye-marking for subsequent same-session VATS resection can be performed, without any delay between diagnosis and treatment. We commonly performed triple dye injection (equal volume of iohexol contrast, methylene blue and indocyanine green) to the lesion via ENB (37). Once dye position has been confirmed by intraoperative CBCT, single lumen endotracheal tube will be exchanged to double lumen tube for VATS. Methylene blue and Indocyanine green can be identified under white light and near-infrared light respectively upon VATS. Optionally, ENB fiducial marker placement to the deep margin of the lesion is an alternative approach to ensure adequate resection margins and optimize oncological outcomes (38). Intra-operative fluoroscopy is used to confirm the position of fiducial marker at the deep margin to allow adequate resecting margin thus optimizing oncological outcomes without sacrificing extra lung volume. These techniques used alone or in combination, allow precise localization of the lesion even if it is too deep for direct visualization or too small and soft in consistency for direct palpation.

In elderly patients who have relatively high surgical risk, or in patients with poor lung function, ablation therapy 


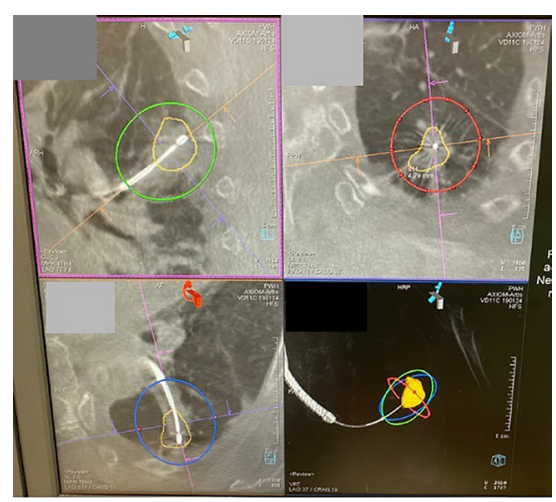

Figure 4 Cone beam CT allows accurate assessment of relationship between bronchoscopic tools and lung lesion. In this case of microwave ablation, the target lung nodule was marked by orange tracings, which was punctured by the ablation catheter. The red, green and blue circles represent the predicted ablation zone, with a minimal margin of $4.3 \mathrm{~mm}$ measured. CT, computed tomography.

is an alternative approach pioneered in Hong Kong for both malignant and pre-malignant lesions (39). Various ablation energies are available on the market, which can be divided into thermal (including radiofrequency ablation, microwave ablation and cryoablation) and nonthermal ablation. Conventional lung ablative treatment via percutaneous approach has shown reasonable result and disease-free survival (37), whereas performing lung ablation via transbronchial route has been a comparatively novel technique. Our institute was one of the first to perform ENB microwave ablation in the HOR. Since 2019, we have completed more than 70 cases of ENB microwave ablation, and the technical success rate was $100 \%$. Complications include pain $(13 \%)$, pneumothorax $(6.7 \%)$ and postablation fever $(6.7 \%)$, which are comparable to or superior to percutaneous lung ablation techniques (40). Advantage of transbronchial ablation when compared to percutaneous ablation is the avoidance of pleural puncture, thus decrease risk of associated complications, like pneumothorax, pleural effusion, bronchopleural fistula and needle tract seeding. Furthermore, ENB ablation has the capability to reach certain areas of lung which are otherwise difficult or dangerous for percutaneous route, for example areas shielded by scapula or close to mediastinum. However, judicious patient selection is important due to the limited ablation zone size of the microwave catheter and the tumor should be away from major blood vessels to prevent heat-sink effect $(40,41)$.

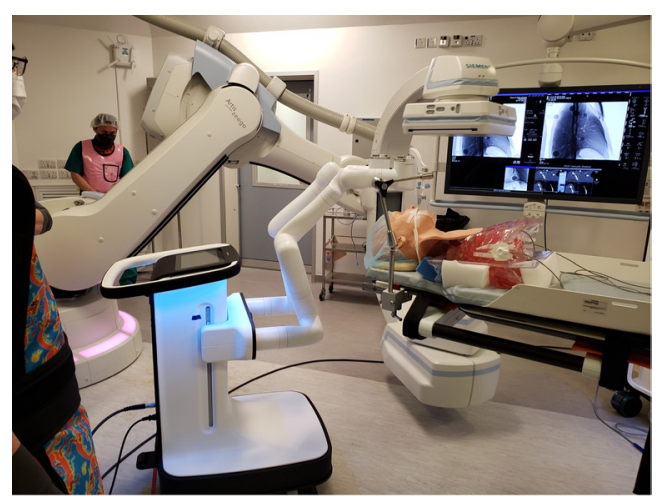

Figure 5 Setup for robotic bronchoscopy in our hybrid operating theatre is shown. The robot consists of two arms holding the bronchoscope in place, which was inserted into the model's airway via an endotracheal tube. An additional metal holder is required to stabilize the entrance of endotracheal tube. The robot is placed at the head side so that CBCT can be performed without obstruction. CBCT, cone-beam computed tomography.

\section{The future of thoracic surgery in Hong Kong}

The use of medical robots in thoracic surgery gained momentum in Hong Kong in recent years. With the refining flexibility of robotic wristed instruments and improved imaging quality of the $3 \mathrm{D}$ thoracoscope, robotic surgery is expected to become more popular and more widely applicable to various thoracic surgical procedures (42). At the present, Da Vinci robotic system (Intuitive, California, USA) has been installed in three out of four thoracic centres in Hong Kong. Common thoracic procedures include robotic thymectomies, excision of posterior mediastinal masses and robotic lobectomies. Competition between robotic companies has provided more choices in the robotic system or platform that best suit our locality and practice. With this increased accessibility to robotic instruments, innovative treatment approaches for thoracic diseases are emerging.

Robotic-assisted bronchoscopy is currently on the horizon for further automation of our ENB procedures with potential for improved accuracy and ergonomics (43) (Figure 6). The bronchoscope is held by robotic arms while surgeons control the advancement of bronchoscope along airways using a remote control. This provides a more stable and exact spatial orientation and thus, aids the operator to perform complex intervention in narrower airways (43). In the future, we foresee the use of robotic-assisted bronchoscopy and trans-bronchial access tools in ENB 


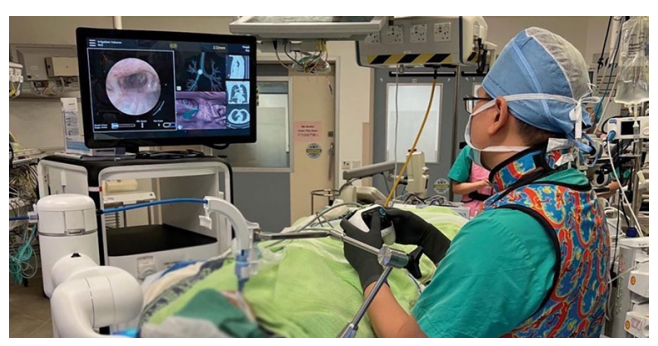

Figure 6 The operator controls the motion of the robotic bronchoscope via a remote controller. On the monitor screen of the console, real-time endobronchial view is shown on the left while virtual bronchoscopy view was shown on the right.

procedure for small peripherally located nodules which otherwise would be challenging to reach. Naturally, the use of different ablative energies is under ongoing research. Alternative energy sources, for example cryoablation and thermal vapor ablation, each has their own specific ablation zone size and shape, and performance characteristic in the lung parenchyma has shown promising early results $(44,45)$. ENB ablative surgery is still in its infancy and will be an abiding research interest in our locality.

The budding technology of magnetic anchored and guided endoscopes (MAGS) developed by the Chinese University of Hong Kong (46) fits perfectly into the pursuit of smaller wounds and less instrument fencing in a cramped port (46). This wireless, high definition videoscope can be delivered into and anchored to the thoracic cavity by an external magnet placed over patient's chest wall. Steerable MAGS is controlled by an external controller which is used to reposition and tilt the videoscope into an ideal position for VATS. This eliminates the space occupied by videoscope through the surgical access, allowing more maneuvering space for other instruments. Additionally, it provides a better, more triangulated plane of vision as compared to traditional thoracoscope. Lastly, the anchoring mechanism offers superior image stabilization during surgery. With the above benefits, MAGS would have a great potential in future minimally invasive thoracic surgery (47).

\section{Conclusions}

As Winston Churchill once said, "To improve is to change; to be perfect is to change often", thoracic surgeons in Hong Kong have been relentless in refining surgical techniques and in exploring innovative solutions, in order to provide the best possible care for patients. In terms of minimal-invasiveness, thoracic surgery has transformed from conventional thoracotomy to multi-portal VATS, and lately to uniportal VATS. In response to a change in disease spectrum, ENB in the hybrid operating room has been developed to provide the perfect one-stop solution to diagnose, localize and treat small and subsolid pre-malignant lesions or early-stage lung cancers. As our citizens have long been recognized for their ingenuity, innovation and resilience; thoracic surgeons in Hong Kong will continue to strive to lead the frontiers of thoracic surgical development.

\section{Acknowledgments}

Funding: The study was supported by Research Grants Council (RGC) University Grant Committee Hong Kong (No. 14119019).

\section{Footnote}

Provenance and Peer Review: This article was commissioned by the Guest Editor (Alan D. L. Sihoe) for the series "Thoracic Surgery Worldwide" published in Fournal of Thoracic Disease. The article has undergone external peer review.

Conflicts of Interest: All authors have completed the ICMJE uniform disclosure form (available at https://jtd.amegroups. com/article/view/10.21037/jtd-21-1600/coif). The series "Thoracic Surgery Worldwide" was commissioned by the editorial office without any funding or sponsorship. CSHN serves as an unpaid editorial board member of fournal of Thoracic Disease from February 2021 to January 2023. and he is a consultant for Johnson and Johnson; Medtronic, USA; and Siemens Healthineer. RWHL is a consultant for Medtronic, USA; and Siemens Healthineer. The authors have no other conflicts of interest to declare.

Ethical Statement: The authors are accountable for all aspects of the work in ensuring that questions related to the accuracy or integrity of any part of the work are appropriately investigated and resolved.

Open Access Statement: This is an Open Access article distributed in accordance with the Creative Commons Attribution-NonCommercial-NoDerivs 4.0 International License (CC BY-NC-ND 4.0), which permits the noncommercial replication and distribution of the article with 
the strict proviso that no changes or edits are made and the original work is properly cited (including links to both the formal publication through the relevant DOI and the license). See: https://creativecommons.org/licenses/by-nc-nd/4.0/.

\section{References}

1. Top Ten Cancers [Internet]. Hong Kong Cancer Registry, Hospital Authority. [updated 2020 Oct; cited 2021 Jul 4]. Available online: https://www3.ha.org.hk/cancereg/ topten.html

2. Chan-Yeung M, Koo LC, Ho JC, et al. Risk factors associated with lung cancer in Hong Kong. Lung Cancer 2003;40:131-40.

3. Yim APC. VATS major pulmonary resection revisitedcontroversies, techniques, and results. Ann Thorac Surg 2002;74:615-23.

4. Yim APC, Ho JKS, Abdullah V. Minimally invasive thoracic surgery: where do we stand now? Hong Kong Med J 1995;1:115-22.

5. Yim AP, Ho JK, Chung SS, et al. One hundred and sixtythree consecutive video thoracoscopic procedures: the Hong Kong experience. Aust N Z J Surg 1994;64:671-5.

6. Yim AP, Izzat MB, Liu HP, et al. Thoracoscopic major lung resections: an Asian perspective. Semin Thorac Cardiovasc Surg 1998;10:326-31.

7. Yim AP, Wan S, Lee TW, et al. VATS lobectomy reduces cytokine responses compared with conventional surgery. Ann Thorac Surg 2000;70:243-7.

8. Ng CS, Lee TW, Wan S, et al. Thoracotomy is associated with significantly more profound suppression in lymphocytes and natural killer cells than video-assisted thoracic surgery following major lung resections for cancer. J Invest Surg 2005;18:81-8.

9. Li WW, Lee RL, Lee TW, et al. The impact of thoracic surgical access on early shoulder function: video-assisted thoracic surgery versus posterolateral thoracotomy. Eur J Cardiothorac Surg 2003;23:390-6.

10. Rocco G, Martucci N, La Manna C, et al. Ten-year experience on 644 patients undergoing single-port (uniportal) video-assisted thoracoscopic surgery. Ann Thorac Surg 2013;96:434-8.

11. Gonzalez D, Paradela M, Garcia J, et al. Single-port videoassisted thoracoscopic lobectomy. Interact Cardiovasc Thorac Surg 2011;12:514-5.

12. Ng CSH. Uniportal video-assisted thoracic surgery: a look into the future. Eur J Cardiothorac Surg 2015;49:i1-i2.

13. Ng CSH, MacDonald JK, Gilbert S, et al. Optimal
Approach to Lobectomy for Non-Small Cell Lung Cancer: Systemic Review and Meta-Analysis. Innovations (Phila) 2019;14:90-116.

14. Harris CG, James RS, Tian DH, et al. Systematic review and meta-analysis of uniportal versus multiportal videoassisted thoracoscopic lobectomy for lung cancer. Ann Cardiothorac Surg 2016;5:76-84.

15. Ng CS, Kim HK, Wong RH, et al. Single-Port VideoAssisted Thoracoscopic Major Lung Resections: Experience with 150 Consecutive Cases. Thorac Cardiovasc Surg 2016;64:348-53.

16. Yu PSY, Chan KW, Lau RWH, et al. Uniportal videoassisted thoracic surgery for major lung resection is associated with less immunochemokine disturbances than multiportal approach. Sci Rep 2021;11:10369.

17. Ng CS, Yeung EC, Wong RH, et al. Single-port sympathectomy for palmar hyperhidrosis with Vasoview Hemopro 2 endoscopic vein harvesting device. J Thorac Cardiovasc Surg 2012;144:1256-7.

18. Chow SCY, Ng CSH. Uniportal Approach to Pericardial Window and Sympathectomy. In: Gonzalez-Rivas D, Ng C, Rocco G, D'Amico T. (eds) Atlas of Uniportal Video Assisted Thoracic Surgery. Springer 2019:51-8.

19. Wang Y, Wong V. Prof. Calvin Sze Hang Ng: always keep an open mind to embrace tomorrow's technologies. J Thorac Dis 2018;10:S1685-7.

20. Journal of Thoracic Disease (dedicated to the 4th Asian Single Port VATS Symposium) 2016;8:S227-S350.

21. European Journal of Cardio-thoracic Surgery (Dedicated to The 3rd Asian Single Port VATS Symposium \& Live Surgery - Redefining the Future of Minimal Invasive Thoracic Surgery. The Chinese University of Hong Kong, 26-27 March 2015) 2016;49:11-i88.

22. Ng CS, Rocco G, Wong RH, et al. Uniportal and singleincision video-assisted thoracic surgery: the state of the art. Interact Cardiovasc Thorac Surg 2014;19:661-6.

23. Ng CS, Wong RH, Lau RW, et al. Minimizing chest wall trauma in single-port video-assisted thoracic surgery. J Thorac Cardiovasc Surg 2014;147:1095-6.

24. Ng CSH, Pickens A, Siegel JM, et al. A novel narrow profile articulating powered vascular stapler provides superior access and haemostasis equivalent to conventional devices. Eur J Cardiothorac Surg 2015;49:i73-i78.

25. Ng CSH, Capili F, Zhao ZR, et al. Laser resection of pulmonary nodule via uniportal thoracoscopic surgery. $\mathrm{J}$ Thorac Dis 2017;9:846-8.

26. Ng CS, Wong RH, Lau RW, et al. Single port videoassisted thoracic surgery: advancing scope technology. Eur 
J Cardiothorac Surg 2015;47:751.

27. Ng CS. Uniportal VATS in Asia. J Thorac Dis 2013;5 Suppl 3:S221-5.

28. Census and Statistics Department of HKSAR. Pattern of Smoking. Hong Kong Monthly Digest of Statistics 2019.

29. Min JH, Lee HY, Lee KS, et al. Stepwise evolution from a focal pure pulmonary ground-glass opacity nodule into an invasive lung adenocarcinoma: an observation for more than 10 years. Lung Cancer 2010;69:123-6.

30. Yu PSY, Chan JWY, Lau RWH, et al. Screening-detected pure ground-glass opacities: malignant potential beyond conventional belief? Transl Lung Cancer Res 2020;9:816-8.

31. Ng CSH, Man Chu C, Kwok MWT, et al. Hybrid DynaCT scan-guided localization single-port lobectomy. Chest 2015;147:1445.

32. Yu PSY, Man Chu C, Lau RWH, et al. Video-assisted thoracic surgery for tiny pulmonary nodules with realtime image guidance in the hybrid theatre: the initial experience. J Thorac Dis 2018;10:2933-9.

33. Chan JW, Yu PS, Lau RW, et al. Hybrid operating roomone stop for diagnosis, staging and treatment of early stage NSCLC. J Thorac Dis 2020;12:123-31.

34. Ng CSH, Yu SCH, Lau RWH, et al. Hybrid DynaCTguided electromagnetic navigational bronchoscopic biopsy. Eur J Cardiothorac Surg 2016;49:i87-i88.

35. Jiang S, Xie F, Mao X, et al. The value of navigation bronchoscopy in the diagnosis of peripheral pulmonary lesions: A meta-analysis. Thorac Cancer 2020;11:1191-201.

36. Folch EE, Pritchett MA, Nead MA, et al. Electromagnetic Navigation Bronchoscopy for Peripheral Pulmonary Lesions: One-Year Results of the Prospective, Multicenter NAVIGATE Study. J Thorac Oncol 2019;14:445-58.

37. $\mathrm{Ng} \mathrm{CSH,} \mathrm{Zhao} \mathrm{Z,} \mathrm{Long} \mathrm{H,} \mathrm{et} \mathrm{al.} \mathrm{Electromagnetic}$ Navigation Bronchoscopy Triple Contrast Dye Marking for Lung Nodule Localization. Thorac Cardiovasc Surg 2020;68:253-5.

Cite this article as: Chang ATC, Chan JWY, Lau RWH, $\mathrm{Ng}$ CSH. Thoracic surgery in Hong Kong. J Thorac Dis 2022;14(8):3075-3082. doi: 10.21037/jtd-21-1600
38. Chan JWY, Lau RWH, Ng CSH. Electromagnetic navigation bronchoscopy fiducial marker margin identification plus triple dye for complete lung nodule resection. JTCVS Tech 2020;3:329-33.

39. Zhao ZR, Lau RWH, Ng CSH. Catheter-based alternative treatment for early-stage lung cancer with a high-risk for morbidity. J Thorac Dis 2018;10:S1864-70.

40. Chan JW, Lau RW, Ngai JC, et al. Transbronchial microwave ablation of lung nodules with electromagnetic navigation bronchoscopy guidance-a novel technique and initial experience with 30 cases. Transl Lung Cancer Res 2021;10:1608-22.

41. Chan JWY, Lau RWH, Ng CSH. Image-Guided Ablative Therapies for Lung Tumors. In: Park HS, editors. Lung Cancer - Modern Multidisciplinary Management. IntechOpen; 2020.

42. Kumar A, Asaf BB. Robotic thoracic surgery: The state of the art. J Minim Access Surg 2015;11:60-7.

43. Agrawal A, Hogarth DK, Murgu S. Robotic bronchoscopy for pulmonary lesions: a review of existing technologies and clinical data. J Thorac Dis 2020;12:3279-86.

44. Steinfort DP, Christie M, Antippa P, et al. Bronchoscopic Thermal Vapour Ablation for Localized Cancer Lesions of the Lung: A Clinical Feasibility Treat-and-Resect Study. Respiration 2021;100:432-42.

45. de Baere T, Tselikas L, Woodrum D, et al. Evaluating cryoablation of metastatic lung tumors In Patients-safety and Efficacy the ECLIPSE Trial-Interim analysis at 1 year. J Thorac Oncol 2015;10:1468-74.

46. Cheng T, Zhang X, Ng CSH, et al. A Novel Magnetic Anchored and Steered Camera Robot for Single Port Access Surgery. 2018 IEEE International Conference on Robotics and Automation (ICRA) 2018:1406-12.

47. Cheng T, Li W, Ng CS, et al. Visual servo control of a novel magnetic actuated endoscope for Uniportal videoassisted Thoracic Surgery. IEEE Robotics and Automation Letters 2019;4:3098-105. 